\title{
Research of Helicoid NC Machining Based on Nine Points Optimization Algorithm
}

\author{
Jiang tong ${ }^{\mathrm{a}}$, Han li, Zhang youjun, Zhang yue \\ School of Mechanical Engineering, Shenyang University Of Technology, Shenyang China \\ a378380402@qq.com
}

Keywords: Helicoid, minimum directed distance, nine point's optimization algorithm, NC machining

\begin{abstract}
Starting with the theory of minimum directed distance, this paper, aiming at the problem of plane programming thought in the NC machining of the helicoids, reaches an important conclusion that the point of minimum directed distance is the meshing point. Besides, this paper achieves a "nine points optimization " algorithm for processing the smooth curve surface, which can effectively calculate the cutter path in multi-coordinate NC machine. On the basis of contract-trace continuity, the adoption of the "nine points optimization " algorithm and tracing prediction method greatly accelerate the computation speed of cutter path.
\end{abstract}

\section{Introduction}

Helicoid is a complicated space curve surface, so if the traditional plane programming method is adopted to calculate cutter location path, the machining accuracy of workpiece will be affected due to large programming error. Nowadays, to find a fast and efficient programming method based on space envelope machining principle has turned into a hot spot in the researching field of helicoids NC machining[1]. At present, in respect of processing screw with the section envelope method, NC programming systems provided by domestic and imported special machines employed at home and abroad adopt plane envelope calculation method, that is, approximately, the contact paths of the cutter and workpiece in the machining process are considered as the plane curves. Its programming thought is to work out that the distance from the end section profile of the workpiece is the equidistance line of the corner radius based on the end section profile of the workpiece; and then based on each point of the equidistance line relative to polar radius of workpiece axis, the cutter location is figured out. In fact, except several special points on the contact trace theoretically, the rest points are approximately obtained, which will surely result in machining error[2].

\section{Theory of minimum directed distance}

Let's hypothesize that there are two continuously differentiable curved surfaces F1 and F2, when F2 moves along the direction of final motion relative to F1, under the circumstance of no interference, the corresponding points of the shortest distance between the two curved surfaces in the direction of motion are the meshing points of the two surfaces[3]. Final motion means the any relative motion between cutter and workpiece is the composition of motion along the each axis in NC machine; therefore, the motion between all cutter locations can be decomposed to be $\mathrm{n}$ independent sequential motions along $\mathrm{m}$ spatial NC axises $(1 \leq \mathrm{n} \leq \mathrm{m})$; usually in the process of continuous processing, it requires that the motion from one cutter location to another one is unilateral and non-redundant; finally, we call the nth independent motion in line with the above-mentioned conditions as final motion; the directed distance indicates $\mathrm{m}$ distances in $\mathrm{NC}$ axises, including straight-line distance and corner distance (see fig.1) 


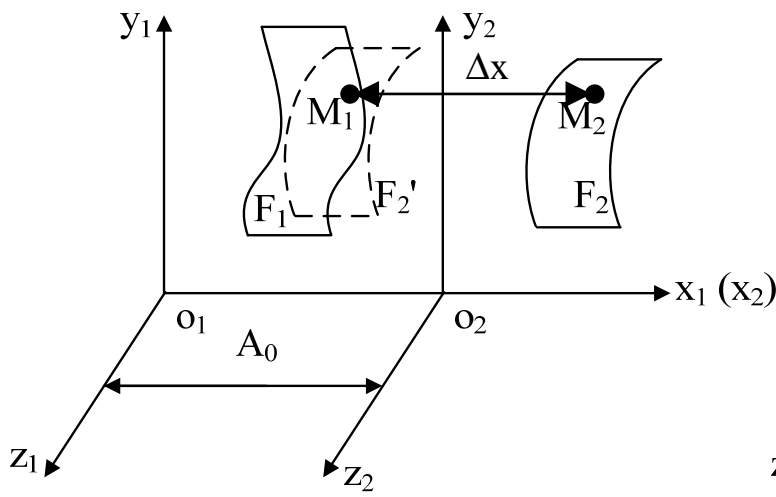

(a) final motion is transactional Fig.1 Theory of Minimum Directed Distance

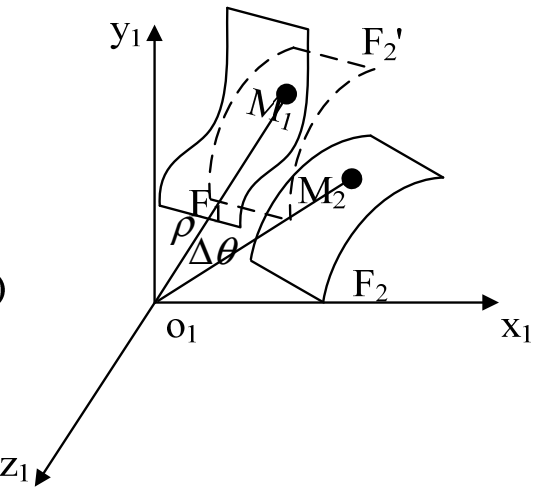

(b final motion is rotational

\section{“Nine points optimization" algorithm}

In the theory of "minimum directed distance", we have already known that finding out the location of every contact point is virtually optimizing each distance function, and the key of optimization problem lies in the selection of the initial point. As is shown in fig. 2 firstly, select a cutter contact point of noninterference on the cutter as the initial point $\mathrm{M}_{0}\left(\alpha_{0}, \beta_{0}\right)$, and calculate the location of next contact point $\mathrm{M}_{1}\left(\alpha_{1}, \beta_{1}\right)$ based on the "minimum directed distance" theory. According to the continuity of contact trace, the points $\mathrm{M}_{0} 、 \mathrm{M}_{1}$ contain the location information of next contact point $\mathrm{M}_{2}$. Mark a Straight line $\mathrm{l}_{1}$ through the points $\mathrm{M}_{0} 、 \mathrm{M}_{1}$, and take the $\Delta \alpha_{1}=\alpha_{1}-\alpha_{0}, \Delta \beta_{1}=\beta_{1}-\beta_{0}$ as step length to get the point $\mathrm{M}_{20}$. Thus, the point $\mathrm{M}_{20}$ is certainly near to the next contact point $\mathrm{M}_{2}$, therefore, it is easy to gain the optimal point in manner of taking $\mathbf{M}_{20}$ as the initial value to calculate the next contact point $\mathbf{M}_{2}$. By analogy, each contact point $\mathbf{M}_{\mathrm{i}+1}$ can be determined by the two previous contact points. With the line cross the two contact points $\mathrm{M}_{\mathrm{i}} 、 \mathrm{M}_{\mathrm{i}-1}$, and $\Delta \alpha_{\mathrm{i}+1}=\alpha_{\mathrm{i}}-\alpha_{\mathrm{i}-1} 、 \Delta \beta_{\mathrm{i}+1}=\beta_{\mathrm{i}}-\beta_{\mathrm{i}-1}$ as the step length, the initial parameter values $\alpha_{\mathrm{i}+1}=\alpha_{\mathrm{i}}+\Delta \alpha_{\mathrm{i}+1}$ 、 and $\beta_{\mathrm{i}+1}=\beta_{\mathrm{i}}+\Delta \beta_{\mathrm{i}+1}$ of the contact point $\mathrm{M}_{\mathrm{i}+1}$ are obtained, and then it approximates the optimal point[4-6].

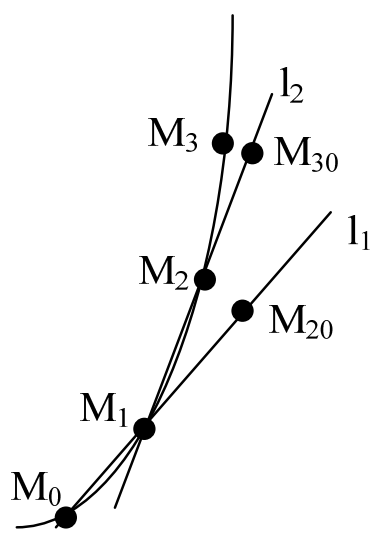

Fig.2 the selection of the initial point

Get the nine points locations (as shown in Fig.3a) by alluding the two curved surfaces $\alpha, \beta$ to a plane. If the point of minimum value is in the centre position 9 (as shown in figure 3a), it indicates that the distance between the corresponding points is greater than it. It reaches the contact in the motion process, thus, its corresponding point in work piece and cutter is the contact point; if the minimum value point locates in the boundary of the nine points (as shown in Fig. 3b and 3c), then recalculate the distance between the curved surface of work piece and the nine points which regards the position of the minimum value as the center, and then judge the location of the minimum value; if it is still in the boundary, extend outward for calculation until the distance between the corresponding 
points around is bigger. If equal distance values appear in the process of distance values comparison, the next approximate center point should be determined by the averages for line and column numbers of the respective positions where the points of equal distance lie. When the average of row number exceeds 1 , take row $=2$; when the average of row number equals to 1 , take row $=0$; when average row value less than 1 , take row $=0$. The column number should be determined in the same way.

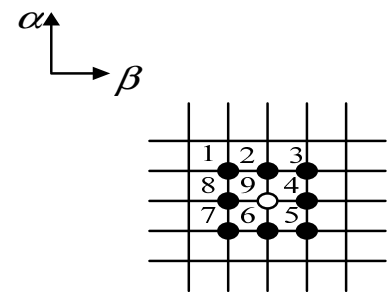

(a)

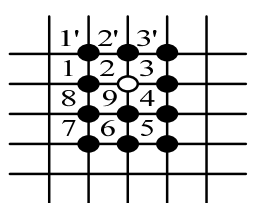

(b)

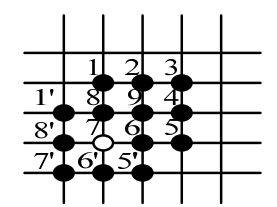

Fig.3 Position of Nine Points

Position of Minimum Points Position of Discrete Points

\section{Application in NC machining}

In production practice, most work pieces have smooth surfaces or piecewise smooth surfaces. The space free-form surface indicated by discrete points can be fitted in curved surface, spline surface, etc, so that it can turn into the smooth surface or piecewise smooth surface. Partial surfaces cut by cutters such as ball-end mill, face cutter with rounding, disc milling cutter, end mill, and so on can be thought of as smooth surfaces. Therefore, the "nine points' optimization" algorithm can be applied to most of the NC programming cases. And its application achieves good effects in screw processing and free-form surface processing. When the continuity of cutter contact path is utilized to calculate the cutter path, more attention should be paid to the smoothness of the work pieces and the cutter. And special treatments at the juncture of piecewise smooth surface is essential to assure the reliability of algorithm.

The example that disc milling cutter with indexable inserts of a tool angle of $35^{\circ}$ processes the 165-type screwdrill rotor produced by a petroleum machinery plant is used to verify the above-mentioned calculation methods. The corner radius is $1.2 \mathrm{~mm}$; cutter diameter $252 \mathrm{~mm}$; and the rotor lead $860 \mathrm{~mm}$. And the parametric equations of end-section profile are as follows:

$$
\left\{\begin{array}{c}
x_{1}=63 \sin (u)-7.5 \sin (6 u)+20 \sin (s) \\
y_{1}=63 \cos (u)-7.5 \cos (6 u)-20 \cos (s)
\end{array}\right.
$$

Where $s=\tan ^{-1}(\sin (5 u) /(1.4-\cos (5 u)))-u$

The parameters of cutter and work-piece are input into the NC automatic programming software which is compiled according to the "nine-point optimization" algorithm; meanwhile let the calculation step be $1^{\circ}$ as well as the center-to-center distance of initial position be A0 $177.7 \mathrm{~mm}$. Therefore, it can work out the cutter path points of a cycle $(\mu=0 \sim 2 \pi / 5)$, as is shown in Fig. 4 .

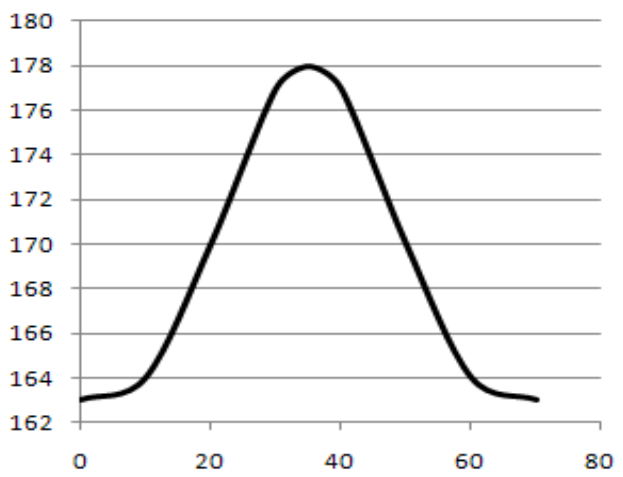

Fig.4 the Cutter Path of Calculation 


\section{Conclusion}

The "nine points optimization" algorithm presented in this paper is of great importance to improve the generating algorithm of cutter location path as well as raise the calculating speed and accuracy. This algorithm is applicable to smooth-faced ball-end mill, face cutter with rounding, disc milling cutter, end mill, etc. This method is also applicable in regular work piece which has smooth surface and piecewise smooth surface. But the junction of piecewise smooth surface needs special treatment. Meanwhile, this algorithm solves the astringency problem in iterative algorithm, and avoids a number of point set calculations in the whole area. Due to its features of fast calculation, simple principal and so on, it turns out to be a very effective method to calculate cutter path in NC machining of complicated curved surface.

\section{Acknowledgements}

This research reported in the paper is financially supported by the Education Office of Liaoning Province (L2010402), the Doctoral Starting up Foundation of Shenyang University of Technology (521101302), Young Backbone Teacher Foundation of Shenyang University of Technology (004436521). These supports are greatly acknowledged.

\section{References}

[1] X.Chen: Journal of Shenyang University of Technology, Vol.32(2010)No.5,pp.520-525.

[2] K.Wang: Journal of Shenyang University of Technology, Vol.32(2010)No.2,pp.162-165.

[3] G.Cao: Chinese Journal of Computational Mechanics,Vol.27(2010)No.5,pp930-935.

[4] Y.Li, X.T.Jiang: Electric Machines and Control,Vol.15(2011)No.6,pp.57-61

[5] X.D.Tong: Journal of Jianghan Petroleum Institute,Vol.27(2005)No.4,pp.713-916

[6] X.C.Zhang: Journal of Shenyang University of Technology Vol.31,n2 (2009), p.173-176 